\title{
New portable tool to screen vestibular and visual function-National Institutes of Health Toolbox initiative
}

\author{
Rose Marie Rine, PT, PhD $;{ }^{1 *}$ Dale Roberts, MS; ${ }^{2}$ Bree A. Corbin, MPT; ${ }^{1}$ Roberta McKean-Cowdin, PhD; ${ }^{3}$ \\ Rohit Varma, MD, MPH; ${ }^{4}$ Jennifer Beaumont, MS $;^{5}$ Jerry Slotkin, PhD $;{ }^{5}$ Michael C. Schubert, PT, PhD ${ }^{\mathbf{6}}$ \\ ${ }^{1}$ University of North Florida, Jacksonville, FL; ${ }^{2}$ Department of Neurology and Neurosurgery, Johns Hopkins Univer- \\ sity School of Medicine, Baltimore, MD; ${ }^{3}$ Department of Preventive Medicine, Keck School of Medicine of University \\ of Southern California, Los Angeles, CA; ${ }^{4}$ Department of Ophthalmology, Keck School of Medicine of University of \\ Southern California, Los Angeles, CA; ${ }^{5}$ Department of Medical Social Sciences, Feinberg School of Medicine, North- \\ western University, Chicago, IL; ${ }^{6}$ Department of Otolaryngology-Head and Neck Surgery, Johns Hopkins University \\ School of Medicine, Baltimore, $M D$
}

\begin{abstract}
As part of the National Institutes of Health Toolbox initiative, we developed a low-cost, easy-to-administer, and time-efficient test of vestibular and visual function. A computerized test of dynamic visual acuity (cDVA) was used to measure the difference in visual acuity between head still and moving in yaw. Participants included 318 individuals, aged 3 to 85 years (301 without and 17 with vestibular pathology). Adults used Early Treatment of Diabetic Retinopathy Study (ETDRS) optotypes; children used ETDRS, Lea, and HOTV optotypes. Bithermal calorics, rotational chair, and light box testing were used to validate the cDVA. Analysis revealed that the cDVA test is reliable for static (intraclass correlation coefficient $[$ ICC] $>/=0.64$ ) and dynamic (ICC $>/=0.43-0.75$ ) visual acuity. Children younger than 6 years old were more likely to complete cDVA with Lea optotypes, but reliability and correlation with ETDRS was better using HOTV optotypes. The high correlation between static acuity and light box test scores $(r=$ 0.795), significant difference of cDVA scores between those with and without pathology $(p</=0.04)$, and the good to excellent sensitivity (73\%) and specificity (69\%) establish that the cDVA is a valid and reliable measure of visual acuity when the head is still and moving, as well as a good proxy of vestibular function to yaw rotation.
\end{abstract}

Key words: dynamic visual acuity, gaze stability, NIH Toolbox, optotype, static visual acuity, vestibular, vestibular rehabilitation, vestibular test, vision, vision test.

\section{INTRODUCTION}

The goal of the National Institutes of Health (NIH) Toolbox initiative is to develop a valid set of instruments that measure motor, sensory, cognitive, and emotive functions for large-scale epidemiological and clinical trials and studies in subjects aged 3 to 85 years [1]. An important mandate of this goal was to develop instruments that could be administered with minimal training

Abbreviations: $\mathrm{BVH}=$ bilateral vestibular hypofunction, cDVA = computerized test of dynamic visual acuity, DVA = dynamic visual activity, E-ETDRS = electronic Early Treatment of Diabetic Retinopathy Study, ENG = electronystagmography, ETDRS = Early Treatment of Diabetic Retinopathy Study, EVA $=$ electronic visual acuity, ICC = intraclass correlation coefficient, NIH = National Institutes of Health, SD = standard deviation, $\mathrm{SVA}=$ static visual acuity, $\mathrm{UVH}=$ unilateral vestibular hypofunction, $\mathrm{VH}=$ vestibular hypofunction, $\mathrm{VOR}=$ vestibular ocular reflex.

*Address all correspondence to Rose Marie Rine, PT, PhD; Specialty Therapy Source, Research, 12948 Palmetto Glade Dr, Jacksonville, FL 32246; 904-762-8419; fax: 904-2215741. Email: specialtytherapy@bellsouth.net http://dx.doi.org/10.1682/JRRD.2010.12.0239 
by nonclinical persons. For the sensory domain, a joint effort was invested by the vestibular and vision function teams to develop a single tool that would be useful for testing both senses. A select group of clinicians and basic scientists ${ }^{*}$ served on separate vestibular and vision teams to identify appropriate tests for their respective sensory area, followed by joint meetings to identify areas of testing overlap between the two teams. The documentation of an increasing need for tests that can identify vestibular and/or visual impairments throughout the lifespan supports the development of tools to meet that need.

The vestibular system is an integral component of human sensory perception, including the perception of angular and linear motion. It provides the brain with both sensory afference and motor efference. Putative vestibular impairments affect 35 percent of U.S. adults over 40 years old [2], with approximately 8 million U.S. adults reporting chronic balance problems [3]. Aside from imbalance, consequences of vestibular deficits also include gaze instability and increased risk for falls [4]. Similar problems exist in pediatric populations. Recently, O’Reilly et al. completed a review of pediatric patients seen over a 4-year period with a chief complaint of dizziness or imbalance [5]. Of those seen by specialists in otolaryngology, 35 percent had confirmed vestibular disorders. Casselbrant et al. reported that balance and vestibular problems persist in children with a history of chronic middle ear effusion after the resolution of the effusion [6]. Together, growing evidence suggests that a significant percentage of adults and children have vestibular deficits, and the incidence in children is underestimated [7-8]. Part of the challenge in identifying the population with vestibular hypofunction $(\mathrm{VH})$ is the existence of a significant time delay from the onset of symptoms to appropriate referral. This is in part caused by the lack of screening tools that can be used by nonspecialists to identify those individuals who should be referred for specialized testing and treatment.

Vision is a complex sensation that provides a personal representation of an individual's surrounding environment. With the aging of the U.S. population, evaluation and treatment of age-related changes in

\footnotetext{
*Vestibular Team: R. M. Rine, M. Schubert, S. Whitney, N. Shepard, D. Wrisley, G. Jacobsen, J. Carey, J. Slotkin, H. Hoffman. Vision Team: R. Varma, R. McKean-Cowdin, K. Cruickshanks, R. Hays, C. Johnson, M. Maguire, R. Massof, C. Owsley, M. Repka, S. Vitale, M. Wall.
}

vision-related diseases will be of growing importance in the next several decades. It is estimated that more than 150 million people in the U.S. general population wear corrective lenses to compensate for visual impairment caused by refractive error [9], which may include myopia, hyperopia, astigmatism, and presbyopia. Other relatively common disease-related causes of visual impairment include age-related macular degeneration, cataracts, diabetic retinopathy, and glaucoma. Studies to prevent and evaluate treatment for these conditions will be of increasing value because the number of people with vision impairment from age-related eye disease is expected to double in the next three decades [9].

Both the vestibular and vision teams were given the task of developing a test that was (1) limited in number of tests and/or demands on the study participants, (2) inexpensive, (3) time efficient, (4) portable, (5) a valid indicator of gaze stability and vision acuity, (6) able to be performed in subjects aged 3 to 85 years, and (7) capable of automated scoring and storing of data. The exclusion criteria for tests to be selected were (1) reliance on selfreport of symptoms, (2) high cost, (3) requirement of examiner expertise, or (4) lack of psychometric strength (i.e., sensitivity, specificity, validity). Based on a comprehensive review of available tests and existing literature, the vestibular team developed two separate tests: one that isolates the vestibular system's contribution to gaze stability (vestibular ocular reflex [VOR]) and one that isolates the vestibular system's contribution to postural control. For the purpose of this article, we will only discuss VOR measurement.

The test chosen for VOR examination was a computerized test of dynamic visual acuity (cDVA) [10-11]. For the vision team, computerized visual acuity testing was also deemed the best way to measure visual acuity. Therefore, the vestibular and vision teams agreed to merge their efforts into a single test of static visual acuity (SVA). While current versions of cDVA are available, they are expensive, require examiner expertise, and have no pediatric correlate. Consequently, efforts in software and hardware development were made to build a new test for use with children and adults that incorporated SVA and dynamic visual acuity (DVA) to evaluate peripheral $\mathrm{VH}$ for the vestibular team and visual resolution for the vision team. 


\section{BACKGROUND}

\section{Dynamic Visual Acuity}

The semicircular canals and otolith organs of the peripheral vestibular end organ are essential for maintaining gaze and gait stability during head motion. This is achieved by efference from the VOR and the vestibulospinal reflexes. Damage to semicircular canals and/or otolith organs causes impaired visual acuity during head rotation (DVA); impaired balance and postural control; and symptoms of vertigo, falling, nausea, and disorientation [10-18]. In addition to these symptoms, children with $\mathrm{VH}$ also experience a progressive motor delay with deleterious consequences on social development and educational performance $[7,19]$. However, lack of availability of low-cost, easy-to-administer tools to screen for VH has limited its identification. The NIH Toolbox cDVA was developed to address this shortcoming.

The DVA test measures the ability to see clearly during head rotation. Scores reflect the difference in visual acuity between stable head and moving head [10-11]. At velocities $<100 \%$, the ability to maintain gaze during head rotation is enabled by visual pursuit and the VOR [20]. For head velocities $>100 \%$, the VOR is the primary contributor for detecting head rotation and maintaining stable gaze [21]. Herdman et al. were the first to develop a cDVA by flashing the letter $\mathrm{E}$ optotype whenever head velocities were between 120 and $180 \%$ s [10]. They presented optotypes in progressively smaller sizes, five at each acuity level, until the subject incorrectly identified five optotypes at a single line of acuity. A rate sensor mounted on a headband worn by the subject monitored active head velocity. The investigators reported good reliability, excellent sensitivity and specificity (94\%), and significant effect of age in both nondisabled controls and patients with VH. In a separate study, Schubert et al. examined the use of a vertical DVA test and also reported excellent reliability (intraclass correlation coefficient $[\mathrm{ICC}]=0.94$ ) for patients with $\mathrm{VH}$, but poor sensitivity in identifying unilateral VH (UVH) loss (ICC = 0.23) [22]. This sensitivity improved to detect bilateral VH (BVH; ICC $=0.55$ ), with the test having excellent specificity to rule out vestibular pathology of any type (ICC $=0.90)$. Each of these aforementioned tests was computerized but limited to testing adults (mean \pm standard deviation $[\mathrm{SD}]=50.6 \pm 17.0$ years) and did not have a validated SVA test.
Rine and Braswell developed a noncomputerized, clinical version of the horizontal DVA test for use with children [23]. Images included Lea symbols (circle, square, house, or apple; Figure 1(a)), posted on a 15-line vision optotype chart [24]. Children aged 3 to 15 years with $(n=11)$ and without $(n=76)$ BVH participated. Subjects initially completed the SVA test [25]. For the DVA test, the child's head was passively moved in the horizontal plane, using a metronome to assure a velocity of $2 \mathrm{~Hz}$. These investigators reported good to excellent reliability (ICC $=0.84$ ) for horizontal DVA and excellent sensitivity and specificity (97\%). However, this study was limited because a significant amount of examiner training was required and the sample was limited to those children with BVH. While UVH is more common than $\mathrm{BVH}$ in adults, we are unaware of the incidence of UVH in children or its effect on DVA in children.

\section{Static Visual Acuity}

SVA tests measure impairments in visual resolution that can be caused by blurring of the retinal image, neural

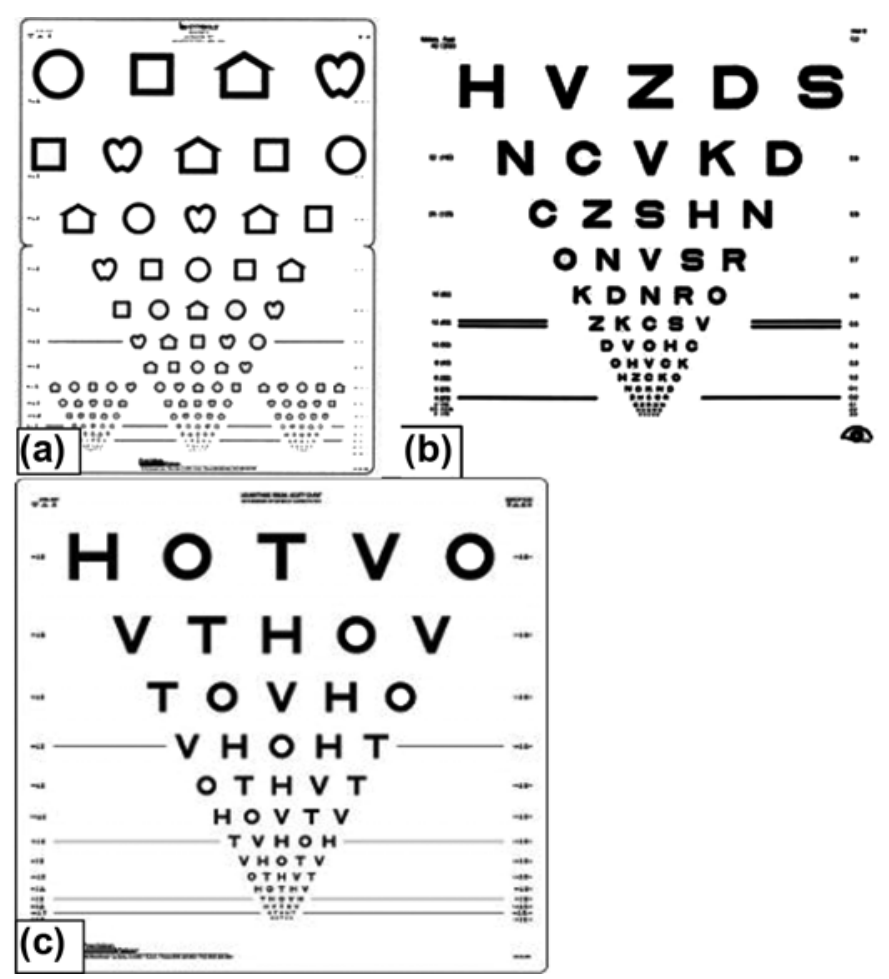

Figure 1.

Vision optotypes used included (a) Lea, (b) Early Treatment of Diabetic Retinopathy Study, and (c) HOTV. 
processing disorders, or damage to neurons in the retina or other parts of the visual pathway. The gold standard for visual acuity testing in adults is the Early Treatment of Diabetic Retinopathy Study (ETDRS) optotype [2627]. The ETDRS optotype was developed to evaluate changes in vision following panretinal photocoagulation in patients with diabetic retinopathy [27]. The test is administered in a standardized way, using visual acuity optotypes illuminated on a light box, resulting in quantitative visual acuity test results. It was designed to allow for comparison of data across research studies and is recommended by the National Academy of Sciences and the American National Standards Institute. The ETDRS optotypes include the same number of letters per row with equal spacing of letters and rows (0.1 logMAR between each row), with the individual rows balanced for letter difficulty. The ETDRS optotyes use 10 letters: C, $\mathrm{D}, \mathrm{H}, \mathrm{K}, \mathrm{N}, \mathrm{O}, \mathrm{R}, \mathrm{S}, \mathrm{V}$, and Z (Figure 1(b)). Scoring is completed on a logarithmic scale.

SVA testing in children (5-12 years old) has traditionally used the letters $\mathrm{H}, \mathrm{O}, \mathrm{T}$, and V (Figure 1(c)), which replaced the ETDRS optotype letters in an attempt to improve testability in younger age groups [28]. Protocols using picture optotypes have also been used with high success rates (e.g., testability) but have been shown to overestimate visual acuity in some children with visual impairment [29]. Several investigators have reported that completion rates are better using symbols, as in the Lea optotypes [25,30], but a concern has been its sensitivity to test amblyopia. The use of HOTV optotypes was standardized in the Amblyopia Treatment Study, during which the protocol was found to have high testability and test-retest reliability in children aged 3 to 7 years [31]. The Amblyopia Treatment Study procedure was modified for an electronic visual acuity (EVA) tester to simplify the testing procedure and decrease testing variability [32]. HOTV testability in developmentally normal children was found to be 100 percent in 5- to 12year-olds but was not tested in younger children. An EVA testing system has also been developed (electronic ETDRS [E-ETDRS] optotype) that uses a testing algorithm that runs on the EVA tester [32-35]. In a study comparing E-ETDRS optotypes with the HOTV optotype, investigators found that children had better test results for HOTV optotypes compared with E-ETDRS optotypes (median difference $=0.06$ logMAR or 3 letters on an optotype with 5 letters per line, $p=0.0001$ ) when including children with normal eyes, eyes with refractive error, or amblyopic eyes [28].

The purpose of this project was to develop an inexpensive, portable, and psychometrically sound measure of visual acuity during still head and head in motion. Specifically, the objectives were to (1) determine the optimal optotype for use with young children, (2) establish the reliability of the DVA and SVA tests, and (3) determine the validity of the DVA and SVA tests.

\section{METHODS}

\section{Subjects}

We enrolled 318 subjects (17 with and 301 without vestibular pathology) in the study. To provide sufficient sample size to allow for repeat testing to determine optimal optotype in children, a larger portion of the sample was aged 12 or younger (Table 1). VH was confirmed by bithermal caloric (adults: >20\% asymmetry) or rotational chair (children: 2 SDs below the normative age-matched data collected in this laboratory) tests. All children completed rotary testing. The types of pathology in this group were BVH secondary to infection $(n=10)$ or mild head trauma $(n=1)$. Only adults with suspect pathology completed bithermal caloric testing to confirm UVH: five caused by viral assault and one deafferented because of removal of vestibular schwannoma. Screening confirmed exclusion criteria, which included preexisting central nervous system disorder, cervical spine pathology, and any oculomotor impairment.

\section{Instrumentation}

Custom software was written in Python and C++. Hardware included a 2 GHz Intel Dual central processing unit laptop with 2 GB of RAM (IBM Thinkpad; Armonk, New York). The laptop was connected to a $1440 \times 900$ resolution monitor that displayed the optotypes. The operator used the built-in laptop display. A single-axis rate sensor (O-Navi; Vista, California) used to detect horizontal head rotation was attached to a soft bicycle light strap and secured to the head (Figure 2). The rate sensor triggered the software to flash an optotype only when head velocity met or exceeded $180 \%$ s. During SVA testing, optotypes were presented one at a time and continuously until the subject identified the optotype (no time limit). During cDVA, the optotype flashed on the screen for 83 ms only when the head moved $180 \%$ s. Optotype 
Table 1.

Subject demographics, $n$ (\%).

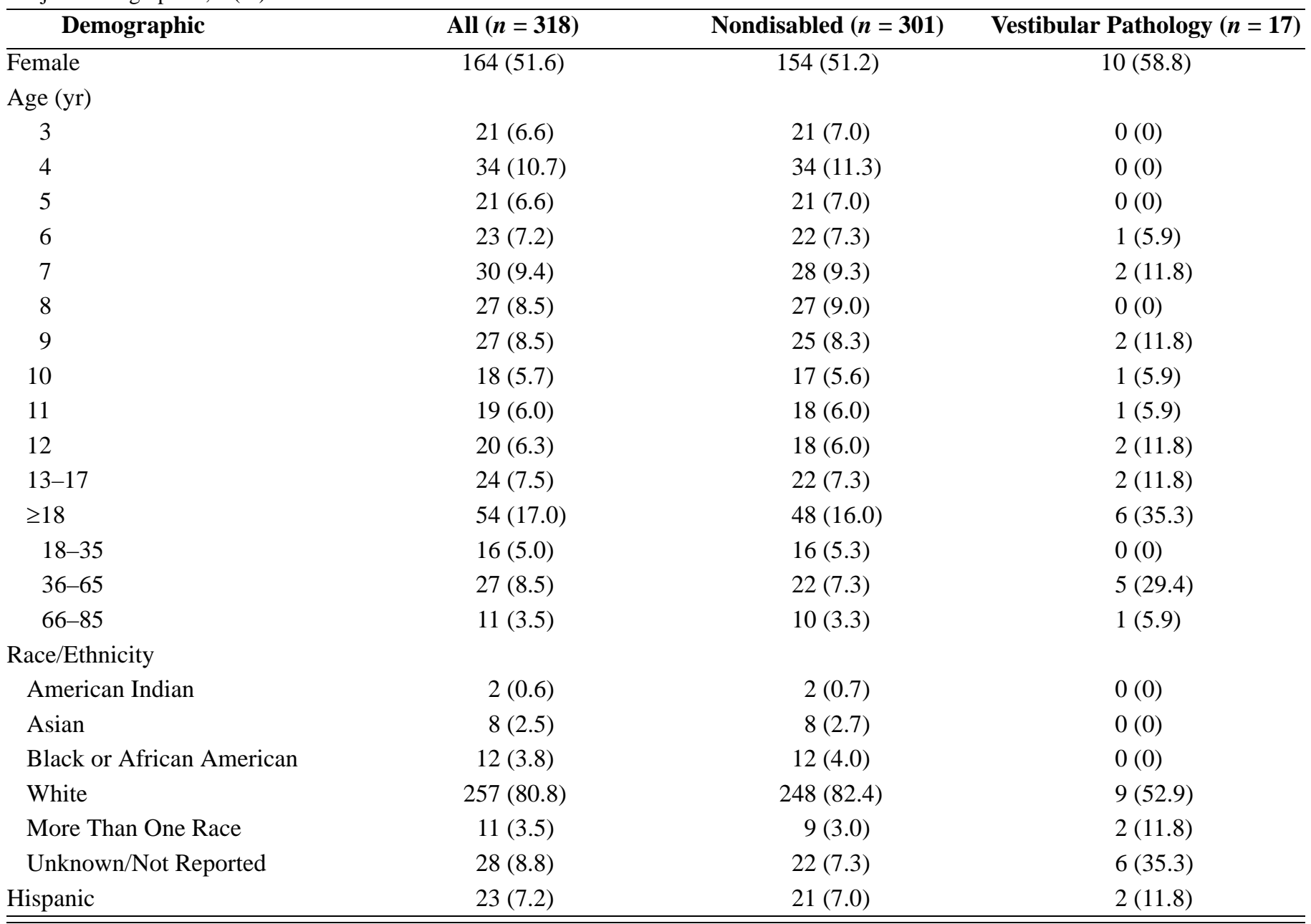

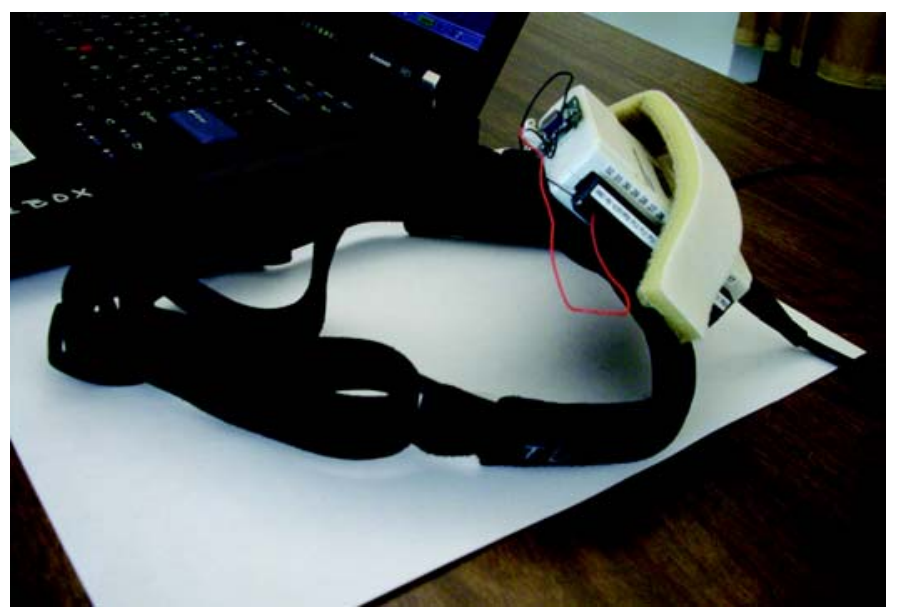

Figure 2.

Dynamic visual acuity headgear. Rate sensor attached to headband. size ranged from 20/10 to 20/800. The software calculated an acuity score (in logMAR) assuming 100 optotypes were viewed using the formula: $1.7-(0.02 \times$ number correct) for SVA and DVA tests. We calculated the cDVA score from these measures by subtracting SVA from cDVA for each side.

\section{Data Collection}

Subjects sat $12.5 \mathrm{ft}$ away from the viewing screen at their eye level. The SVA test begins with an initial "quick" screening at size 20/50 and presents a single random letter per size (in steps of 0.1 logMAR), either going smaller until one is identified incorrectly or larger until one is identified correctly. The test then presents five letters per line size, beginning with the smallest size answered correctly during screening. If any letter is missed at the largest size seen, the next largest level is added. If three 
or more letters are correct at the smallest size seen, the next smallest level is added. The subject always sees all five letters for each line size. Letters from the largest level in the test are always shown first, working down to the smallest level. Testing stops when three or more letters are missed at the smallest level or the subject reaches the 20/10 level. The DVA test follows the same procedure, except it avoids the screening portion and starts three line sizes larger than the SVA screening. However, we performed training trials to assure correct head movement. The operator enters the subject's responses. The software records the history of all optotypes displayed and the participants' responses.

Testing was performed first with the head still (SVA) and again with the head moving (DVA) to the right (two trials) and then to the left (one trial). The first trial with rotation to the right was considered training for all subjects. We discarded scores from this trial and used them to control for learning effect [10-11].

Subjects self-generated the horizontal head rotations and were instructed to "move your head side to side as if saying no." Children completed training trials for DVA testing until they obtained 80 percent accuracy (4/5 correct optotypes). Young children responded verbally or by pointing to a card with the optotypes on it (at a size of 20/ $100)$. Subjects $(n=299)$ completed each test sequence twice to examine reliability.

For all subjects over 12 years old, we used the ETDRS optotype. To determine the optimal optotype set for use with children, we used three optotype sets (Lea, HOTV, and ETDRS); for subjects aged 3 to 6 years, we used both Lea symbols and HOTV optotypes, and for subjects aged 7 to 12 years, we completed tests using all three optotypes. Presentation sequence of optotypes for these groups was performed using a random block design.

To encourage participation by the youngest children, the examiners used reward stickers. Often, young children tend to avoid objects placed on their head, so the examiners offered "hats" (e.g., crown, pirate hat, or themed baseball cap) that could be attached to the head gear (Figure 3). Children were able to take their hat with them when testing was complete.

ETDRS light box acuity testing was completed by all subjects $\geq 7$ years old to examine validity of the computerized SVA test. We asked subjects to read each letter of an ETDRS optotype placed $12.5 \mathrm{ft}$ away, backlit with a light box. The test was complete when the subject missed three out of five optotypes at a line size (in logMAR) or

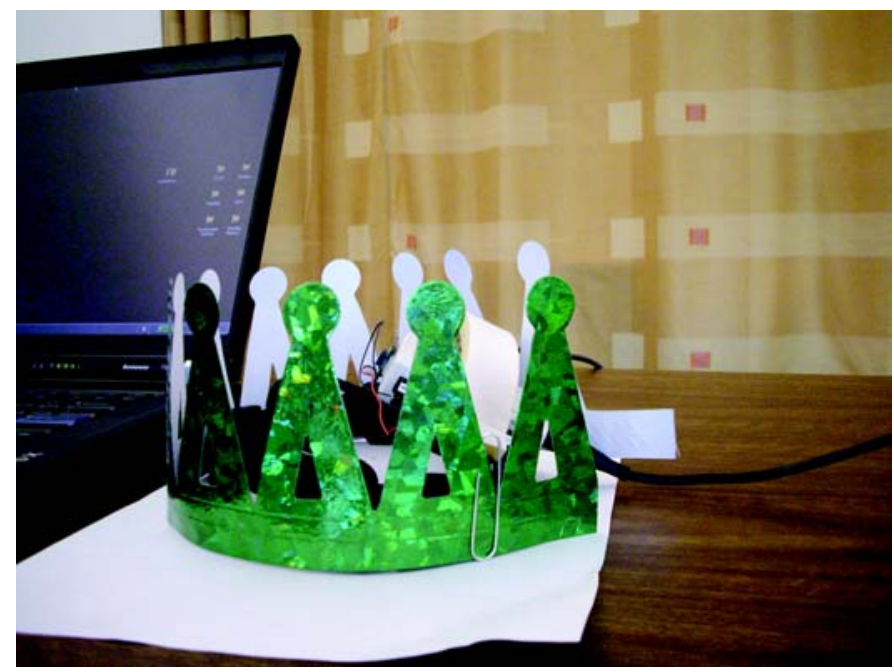

Figure 3.

Hat incentive for children. To encourage participation and wearing rate sensor, paper hats were attached to headband. Rate sensor was concealed to minimize any anxiety and curiosity.

read the entire optotype [27]. The SVA score was the line size (logMAR) just above this.

\section{Statistical Analysis}

We estimated test-retest reliability using ICCs within each of the age groups. To evaluate agreement between acuity scores based on different optotypes and tests, we computed ICCs between the pairs of measures (Lea, HOTV, ETDRS) within each age group and collapsed across age groups. We evaluated usability of each measure among children by examining the number able to successfully complete each test. We examined the associations of each measure with the relevant gold standard. For children, we used data from the rotational chair as a criterion reference; for adults, we used electronystagmography (ENG) as the reference to validate those subjects with vestibular pathology. In addition, we used a prior-established cDVA [36] to establish validity (sensitivity and specificity) in adults. For children, we used rotary test results to establish validity. To compare cDVA scores between those who did and did not pass the gold standard examination (evaluate construct validity), we used two-sample $t$-tests. We calculated effect sizes (mean difference divided by pooled SD) for all group comparisons to aid interpretation. 


\section{RESULTS}

\section{Demographics and Descriptives}

Of the sample, 52 percent was female and 7 percent was Hispanic (Table 1). Of the 318 total participants, a small sample $(n=17)$ had a diagnosed vestibular pathology. Most of the individuals with vestibular pathology (14/17; $82 \%$ ) were $\geq 9$ years old.

Completion rates differed in the youngest groups (Table 2). For SVA testing, children 3 to 4 years old more successfully completed the test using the Lea optotypes instead of the HOTV optotypes.

\section{Reliability}

The reliability of SVA testing for each optotype depended on the optotype used but ranged from excellent to good (ICC $=0.89,0.91$, and 0.84 for Lea, HOTV, and ETDRS, respectively; Table 3). Reliability for cDVA was acceptable for the HOTV and ETDRS optotypes but not the Lea optotype (ICC $=0.55$ for HOTV, 0.65 for ETDRS, 0.43 for Lea; Table 3). The difference in reli- ability of the optotypes was more pronounced in DVA testing (Table 3).

The following correlation of SVA scores between optotypes existed: Lea and HOTV = moderate to excellent $(r=0.58-0.98$; overall $r=0.88)$; Lea and ETDRS = fair to excellent ( $r=0.41-0.96$; overall $r=0.90)$, and HOTV and ETDRS = good to excellent $(r=0.72-0.97$; overall $r=0.93$ ).

\section{Validity}

Sensitivity and specificity of cDVA was fair to excellent (calculations based on ETDRS optotypes only $[n=$ 210]; Table 4). For children, scores achieved by those with and without $\mathrm{VH}$ were significantly different $(p \leq$ 0.001 ; effect size $=0.7$; Figure 4). In adults, those who failed the previously established cDVA differed significantly from those who did not $(p \leq 0.001$; effect size $=$ 1.9; Figure 4). The agreement between the computerized version of SVA and light box visual acuity scores was $\operatorname{good}(r=0.795 ; p \leq 0.001$; Figure 5).

Table 2.

Success rates for completion of testing by age and optotype. All other age groups achieved 100 percent completion rates.

\begin{tabular}{|c|c|c|c|c|}
\hline \multirow{2}{*}{ Age (yr) } & \multicolumn{2}{|c|}{ Static Visual Acuity (\%) } & \multicolumn{2}{|c|}{ Dynamic Visual Acuity (\%) } \\
\hline & Lea & HOTV & Lea & HOTV \\
\hline 3 & 81 & 76 & 70 & 40 \\
\hline 4 & 94 & 86 & 85 & 50 \\
\hline
\end{tabular}

Table 3.

Reliability of visual acuity measures.

\begin{tabular}{|c|c|c|c|c|c|c|}
\hline \multirow[b]{2}{*}{ Measure } & \multicolumn{6}{|c|}{ ICC (95\% CI) } \\
\hline & All & $\begin{array}{c}\text { Age } 3-4 \\
(n=37 \text { to } 50)\end{array}$ & $\begin{array}{c}\text { Age } 5-6 \\
(n=43 \text { to } 44)\end{array}$ & $\begin{array}{c}\text { Age } 7-12 \\
(n=133 \text { to } 141)\end{array}$ & $\begin{array}{c}\text { Age 13-17 } \\
(n=24)\end{array}$ & $\begin{array}{c}\text { Age } 18-76 \\
(n=50 \text { to } 54)\end{array}$ \\
\hline Static Visual Acuity & & & & & & \\
\hline Lea & $0.89(0.86,0.92)$ & $0.64(0.35,0.82)$ & $0.75(0.53,0.87)$ & $0.92(0.89,0.94)$ & - & - \\
\hline HOTV & $0.91(0.88,0.93)$ & $0.73(0.47,0.87)$ & $0.61(0.32,0.80)$ & $0.93(0.90,0.95)$ & - & - \\
\hline ETDRS & $0.84(0.79,0.88)$ & - & - & $0.85(0.80,0.89)$ & $0.74(0.50,0.88)$ & $0.81(0.62,0.91)$ \\
\hline Lea (right) & $0.29(0.15,0.42)$ & $0.09(0,0.46)$ & $0.50(0.16,0.73)$ & $0.22(0.05,0.37)$ & - & - \\
\hline Lea (average) & $0.43(0.31,0.54)$ & $0.32(0,0.63)$ & $0.52(0.19,0.74)$ & $0.34(0.18,0.48)$ & - & - \\
\hline HOTV (left) & $0.43(0.30,0.54)$ & $0.57(0.21,0.79)$ & $0.63(0.35,0.81)$ & $0.28(0.12,0.43)$ & - & - \\
\hline HOTV (right) & $0.46(0.34,0.57)$ & $0.46(0.06,0.73)$ & $0.18(0,0.51)$ & $0.46(0.32,0.58)$ & - & - \\
\hline HOTV (average) & $0.55(0.44,0.64)$ & $0.63(0.30,0.83)$ & $0.51(0.18,0.74)$ & $0.48(0.34,0.60)$ & - & - \\
\hline
\end{tabular}


JRRD, Volume 49, Number 2, 2012

Table 4.

Sensitivity and specificity (using Early Treatment of Diabetic Retinopathy Study optotype) of computerized test of dynamic visual acuity (cDVA).

\begin{tabular}{lrrr}
\hline \multirow{2}{*}{ cDVA } & \multicolumn{3}{c}{ Pathology $(\boldsymbol{n})$} \\
\cline { 2 - 4 } & No & Yes & Total \\
\hline Pass & 135 & 4 & 139 \\
Fail & 60 & 11 & 71 \\
Total & 195 & 15 & 210 \\
\hline Not:
\end{tabular}

Note: Sensitivity $=73 \%$; specificity $=69 \%$.

\section{DISCUSSION}

The cDVA created for the NIH Toolbox initiative is a valid measure of SVA, DVA, and VH. For children, cDVA reliability is moderate when using the ETDRS optotype and fair when using the Lea and HOTV optotypes. This might be because of the minimal variation in scores, since most subjects with pathology were $>9$ years old and thus were tested using only the ETDRS optotype. cDVA is a functional measure of VOR and will not replace the current standard of clinical vestibular function testing (i.e., bithermal caloric or rotary tests with
ENG). This is in part because of the test being performed using active head rotations, which are known to recruit strategies to assist gaze stability, different from the VOR [37]. However, our version of the cDVA would be a useful means to screen visual acuity and VOR, particularly in large population-based studies.

Similar to prior reports $[25,30]$, we had more success using the Lea optotypes for children $<5$ years old. This was particularly evident in DVA testing, which may be because of the increased difficulty of the dual task of moving the head and identifying a symbol. However, scores from HOTV had a higher correlation with the ETDRS

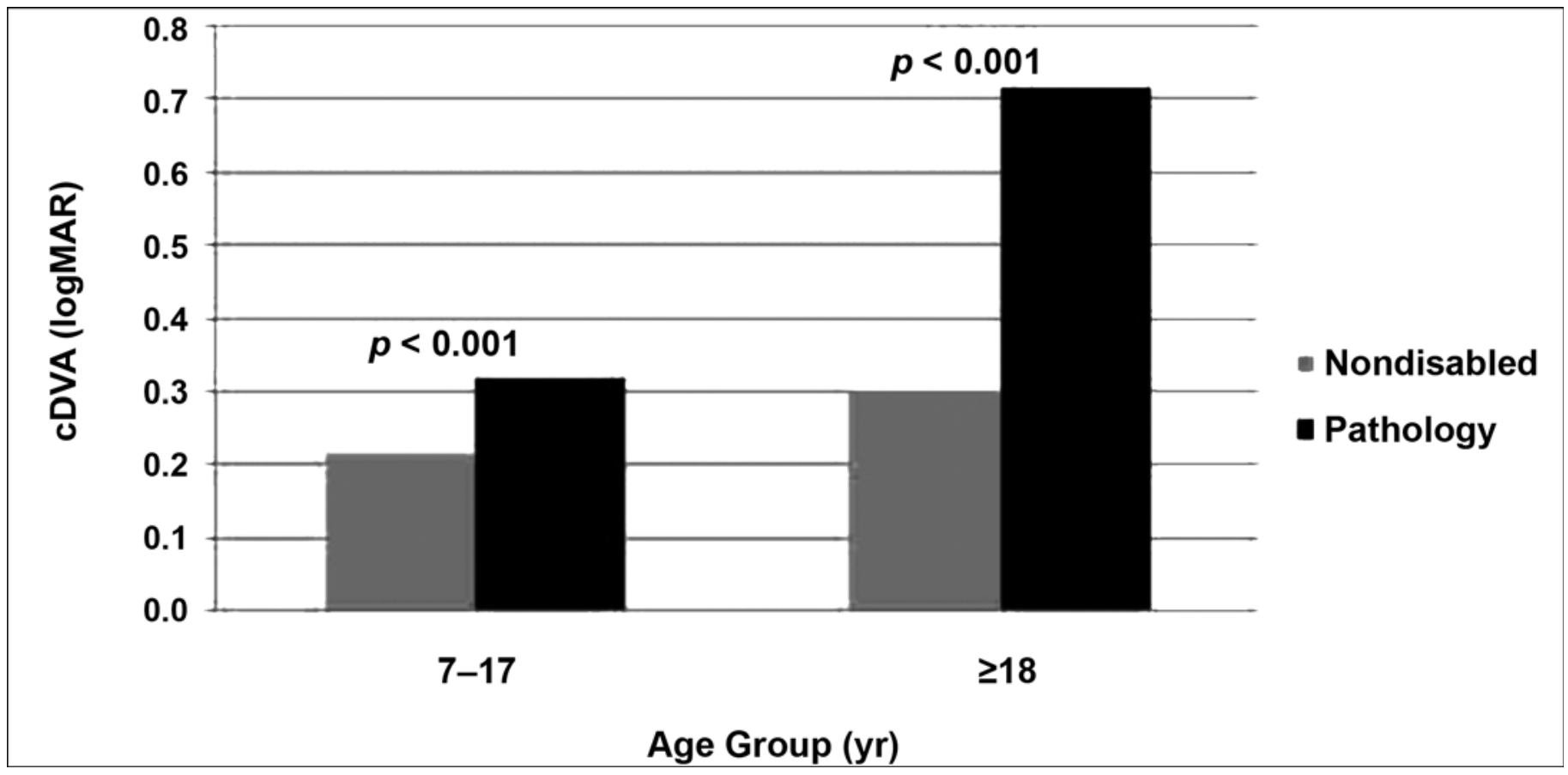

Figure 4.

Comparison of computerized testing of dynamic visual acuity (CDVA) score between those with and without pathology. cDVA score (dynamic visual acuity score - static visual acuity score, in logMAR) differed significantly between those with and without pathology in both younger and older age groups. 


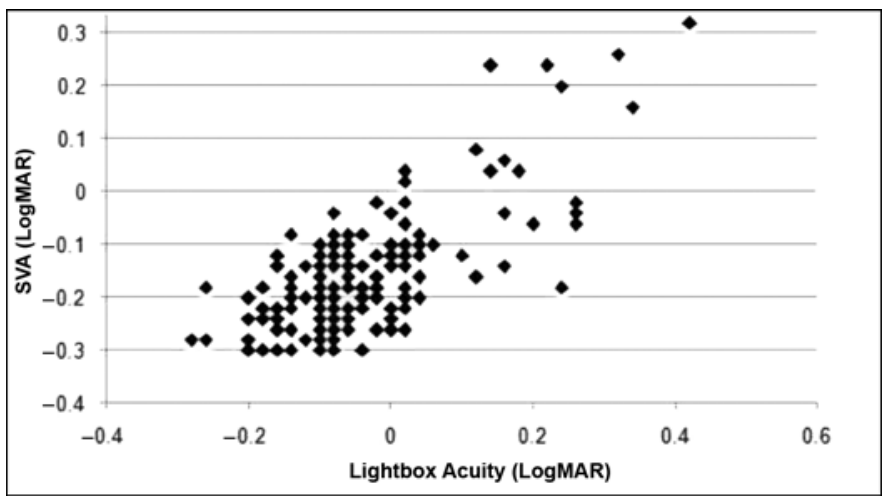

Figure 5.

Correlation of computerized static visual acuity (SVA) and light box testing scores. SVA scores (in logMAR) attained on computerized test and light box test were highly correlated $(r=$ $0.795 ; p<0.001)$.

optotype. These results suggest that the ideal optotype for children $<6$ years old is the HOTV optotype for SVA. However, if cDVA is of primary interest to investigators, the Lea optotype is optimal for success rates with very young children. Some of the challenges we experienced that influenced testability in children were that they were not comfortable or secure identifying letters, they were concerned they had made an error (or at least felt they did and did not want to continue), or they became bored with the task. All children were comfortable identifying the symbols and thus did not feel challenged until the optotype became small. With a dual discomfort level (letters and size with HOTV and ETDRS optotypes), children often wanted to stop all testing, with participation in the dynamic component adding a third challenge. Therefore, depending on the purpose of the data, cDVA in children should use the optotype that affords the most success (e.g., Lea optotype). Note that for this study, children completed testing of two or three different optotypes. This would not be the case in the typical clinical setting. In our experience, success in testing young children is maximized by keeping an upbeat, positive approach and using plenty of encouragement, applauding all responses.

We were able to complete the cDVA within 5 min for most subjects. Occasionally, testing in younger children required more demonstration, breaks, and rests and could thus require $7 \mathrm{~min}$. This may be greatly influenced by the skill of the investigator working with children even though the protocol is computerized.
Both vestibular and visual functions are expected to be critical components of the functional status of individuals of all ages. This is primarily because of the growing evidence of vestibular and visual impairments in adults and children [2-3,5,7,38]. As we age, vestibular afferent neurons die and function similarly becomes impaired [39]. Refractive error is also a common condition in the general population, and the level of visual impairment caused by age-related diseases is expected to increase in the general population over the next several decades as the age distribution shifts toward greater numbers of individuals in older age groups. A readily available, inexpensive tool that enables screening of visual acuity and vestibular function by individuals with minimal training is important for healthcare and research.

\section{LIMITATIONS}

The limited number of subjects with vestibular pathology younger than 6 years old limits the generalization of sensitivity and specificity reported to those older than 6 years old. This, and the lack of gold standard testing of vestibular function in adults without vestibular pathology, may have affected results presented here. Furthermore, most subjects had visual acuity at or above 20/ 20. Further study is warranted that includes individuals with vestibular pathology across all ages, with gold standard testing of vestibular function of those with and without vestibular pathology, and those with visual acuity impairments to comprehensively establish the validity of this test.

\section{CONCLUSIONS}

Screening for vestibular function and visual acuity requires specialized training and expensive equipment that is not typically portable. Based on results presented here, the cDVA developed for use in the NIH Toolbox yields reliable and valid measures of both SVA and DVA. The test is inexpensive, portable, and can be completed by individuals aged 3 to 85 years. No special expertise in visual or vestibular testing is needed, and the test can be completed within $5 \mathrm{~min}$. This makes it an optimal tool to screen for vestibular functional status by clinicians in various settings and investigators alike. Continuing research 
is underway to establish a normative database for individuals aged 3 to 85 years.

\section{ACKNOWLEDGMENTS}

\section{Author Contributions:}

Study concept and design: R. M. Rine, M. C. Schubert, D. Roberts, J. Slotkin, R. McKean-Cowdin, R. Varma.

Acquisition of data: R. M. Rine, M. C. Schubert, B. A. Corbin, R. McKean-Cowdin.

Analysis and interpretation of data: R. M. Rine, M. C. Schubert, J. Beaumont, R. Varma.

Drafting of manuscript: R. M. Rine, M. C. Schubert, D. Roberts, J. Slotkin, R. McKean-Cowdin, R. Varma, B. A. Corbin, J. Beaumont. Critical revision of manuscript for important intellectual content:

R. M. Rine, M. C. Schubert, J. Slotkin, R. McKean-Cowdin, R. Varma. Statistical analysis: J. Beaumont.

Administrative, technical, or material support: D. Roberts, B. A. Corbin, M. C. Schubert.

Study supervision: J. Slotkin, R. M. Rine, M. C. Schubert, R. Varma. Financial Disclosures: The authors have declared that no competing interests exist.

Funding/Support: This material was based on work supported in part by the Blueprint for Neuroscience Research and the Basic Behavioral and Social Science Opportunity Network (OppNet), NIH (grant HHSN-260-2006-00007-C).

Additional Contributions: Dr. Rine is now with Specialty Therapy Source, Jacksonville, Florida.

Institutional Review: We obtained informed consent for all subjects 18 years and older, parental consent for all minors, and assent for all 7 to 17 year olds (as approved by the institutional review boards of the University of North Florida, Johns Hopkins University, and University of Southern California).

Participant Follow-Up: The authors do not plan to inform participants of the publication of this study due to a lack of contact information. However, participants were informed that the results would be submitted for publication and encouraged to check the study Web site for an updated list of publications.

\section{REFERENCES}

1. Gershon RC, Cella D, Fox NA, Havlik RJ, Hendrie HC, Wagster MV. Assessment of neurological and behavioural function: The NIH Toolbox. Lancet Neurol. 2010;9(2): 138-39. [PMID: 20129161] http://dx.doi.org/10.1016/S1474-4422(09)70335-7

2. Agrawal Y, Carey JP, Della Santina CC, Schubert MC, Minor LB. Disorders of balance and vestibular function in US adults: Data from the National Health and Nutrition Examination Survey. Arch Intern Med. 2009;169(10):938-44. [PMID: 19468085] http://dx.doi.org/10.1001/archinternmed.2009.66
3. NIH health disparities strategic research plan and budget fiscal years 2009-2013. Bethesda (MD): National Institute of Deafness and Communication Disorders; 2010.

4. Wei D, Hain TC, Proctor LR. Head-shaking nystagmus: Associations with canal paresis and hearing loss. Acta Otolaryngol. 1989;108(5-6):362-67. [PMID: 2589066] http://dx.doi.org/10.3109/00016488909125540

5. O’Reilly RC, Morlet T, Nicholas BD, Josephson G, Horlbeck D, Lundy L, Mercado A. Prevalence of vestibular and balance disorders in children. Otol Neurotol. 2010;31(9): 1441-44. [PMID: 20729773]

6. Casselbrant ML, Furman JM, Mandel EM, Fall PA, KursLasky M, Rockette HE. Past history of otitis media and balance in four-year-old children. Laryngoscope. 2000;110(5): 773-78. [PMID: 10807356] http://dx.doi.org/10.1097/00005537-200005000-00007

7. Rine RM. Growing evidence for balance and vestibular problems in children. Audiol Med. 2009;7(3):138-42. http://dx.doi.org/10.1080/16513860903181447

8. Worden BF, Blevins NH. Pediatric vestibulopathy and pseudovestibulopathy: Differential diagnosis and management. Curr Opin Otolaryngol Head Neck Surg. 2007; 15(5):304-9. [PMID: 17823544] http://dx.doi.org/10.1097/MOO.0b013e3282bf139e

9. Friedman DC, Congdon N, Kempen J, Tielsch JM. Vision problems in the U.S.: Prevalence of adult vision impairment and age-related eye disease in America. Bethesda (MD): National Eye Institute; 2008.

10. Herdman S, Tusa RJ, Blatt P, Suzuki A, Venuto PJ, Roberts D. Computerized dynamic visual acuity test in the assessment of vestibular deficits. Am J Otol. 1998;19(6):790-96. [PMID: 9831156]

11. Schubert MC, Herdman SJ, Tusa RJ. Functional measure of gaze stability in patients with vestibular hypofunction. Ann N Y Acad Sci. 2001;942:490-91. [PMID: 11710496] http://dx.doi.org/10.1111/j.1749-6632.2001.tb03777.x

12. Grossman GE, Leigh RJ. Instability of gaze during locomotion in patients with deficient vestibular function. Ann Neurol. 1990;27(5):528-32. [PMID: 2360793] http://dx.doi.org/10.1002/ana.410270512

13. Grossman GE, Leigh RJ, Bruce EN, Huebner WP, Lanska DJ. Performance of the human vestibuloocular reflex during locomotion. J Neurophysiol. 1989;62(1):264-72. [PMID: 2754477]

14. Brookhouser PE, Cyr DG, Peters JE, Schulte LE. Correlates of vestibular evaluation results during the first year of life. Laryngoscope. 1991;101(7 Pt 1):687-94.

[PMID: 2062147]

15. Hillman EJ, Bloomberg JJ, McDonald PV, Cohen HS. Dynamic visual acuity while walking in normals and labyrinthine-deficient patients. J Vestib Res. 1999;9(1):49-57. [PMID: 10334016] 
16. Mirka A, Black FO. Clinical application of dynamic posturography for evaluating sensory integration and vestibular dysfunction. Neurol Clin. 1990;8(2):351-59.

[PMID: 2193216]

17. Allum JH, Bloem BR, Carpenter MG, Honegger F. Differential diagnosis of proprioceptive and vestibular deficits using dynamic support-surface posturography. Gait Posture. 2001;14(3):217-26. [PMID: 11600325] http://dx.doi.org/10.1016/S0966-6362(01)00142-4

18. Dichigans J, Deiner HC. The use of short- and long-latency reflex testing in leg muscles of neurological patients. In: Struppler A, Weindl A, editors. Clinical aspects of sensory motor integration. New York (NY): Springer-Verlag; 1987. p. $165-75$. http://dx.doi.org/10.1007/978-3-642-71540-2_19

19. Braswell J, Rine RM. Evidence that vestibular hypofunction affects reading acuity in children. Int J Pediatr Otorhinolaryngol. 2006;70(11):1957-65. [PMID: 16945429] http://dx.doi.org/10.1016/j.ijporl.2006.07.013

20. Meyer CH, Lasker AG, Robinson DA. The upper limit of human smooth pursuit velocity. Vision Res. 1985;25(4): 561-63. [PMID: 4060608] http://dx.doi.org/10.1016/0042-6989(85)90160-9

21. Lee MH, Durnford SJ, Crowley JS, Rupert AH. Visual vestibular interaction in the dynamic visual acuity test during voluntary head rotation. Aviat Space Environ Med. 1997;68(2): 111-17. [PMID: 9125086]

22. Schubert MC, Herdman SJ, Tusa RJ. Vertical dynamic visual acuity in normal subjects and patients with vestibular hypofunction. Otol Neurotol. 2002;23(3):372-77. [PMID: 11981398] http://dx.doi.org/10.1097/00129492-200205000-00025

23. Rine RM, Braswell J. A clinical test of dynamic visual acuity for children. Int J Pediatr Otorhinolaryngol. 2003;67(11): 1195-1201. [PMID: 14597370] http://dx.doi.org/10.1016/j.ijporl.2003.07.004

24. Lea symbols vision chart [Internet]. Elgin (IL): Good-lite Co; 2010. Available from: https://www.good-lite.com/default.cfm

25. Becker R, Hübsch S, Gräf MH, Kaufmann H. Examination of young children with Lea symbols. Br J Ophthalmol. 2002; 86(5):513-16. [PMID: 11973243]

http://dx.doi.org/10.1136/bjo.86.5.513

26. Bailey IL, Lovie JE. New design principles for visual acuity letter charts. Am J Optom Physiol Opt. 1976;53(11): 740-45. [PMID: 998716]

27. Ferris FL 3rd, Kassoff A, Bresnick GH, Bailey I. New visual acuity charts for clinical research. Am J Ophthalmol. 1982;94(1):91-96. [PMID: 7091289]

28. Rice ML, Leske DA, Holmes JM. Comparison of the amblyopia treatment study HOTV and electronic-Early Treatment of Diabetic Retinopathy Study visual acuity protocols in children aged 5 to 12 years. Am J Ophthalmol.
2004;137(2):278-82. [PMID: 14962417]

http://dx.doi.org/10.1016/j.ajo.2003.08.016

29. Mayer DL, Gross RD. Modfied Allen pictures to assess amblyopia in young children. Ophthalmology. 1990;97(6): 827-32. [PMID: 2374689]

30. Repka MX. Use of Lea symbols in young children. $\mathrm{Br} \mathrm{J}$ Ophthalmol. 2002;86(5):489-90. [PMID: 11973238] http://dx.doi.org/10.1136/bjo.86.5.489

31. Holmes JM, Beck RW, Repka MX, Leske DA, Kraker RT, Blair RC, Moke PS, Birch EE, Saunders RA, Hertle RW, Quinn GE, Simons KA, Miller JM; Pediatric Eye Disease Investigator Group. The amblyopia treatment study visual acuity testing protocol. Arch Ophtalmol (Paris). 2001;119(9): 1345-53. [PMID: 1154641$]$

32. Moke PS, Turpin AH, Beck RW, Holmes JM, Repka MX, Birch EE, Hertle RW, Kraker RT, Miller JM, Johnson CA. Computerized method of visual acuity testing: Adaptation of the amblyopia treatment study visual acuity testing protocol. Am J Ophthalmol. 2001;132(6):903-9.

[PMID: 11730656]

http://dx.doi.org/10.1016/S0002-9394(01)01256-9

33. Beck RW, Moke PS, Turpin AH, Ferris FL 3rd, SanGiovanni JP, Johnson CA, Birch EE, Chandler DL, Cox TA, Blair RC, Kraker RT. A computerized method of visual acuity testing: Adaptation of the Early Treatment of Diabetic Retinopathy Study testing protocol. Am J Ophthalmol. 2003;135(2):194-205. [PMID: 12566024$]$ http://dx.doi.org/10.1016/S0002-9394(02)01825-1

34. Cotter SA, Chu RH, Chandler DL, Beck RW, Holmes JM, Rice ML, Hertle RW, Birch EE, Moke PS. Reliability of the electronic Early Treatment Diabetic Retinopathy Study testing protocol in children 7 to $<13$ years old. Am J Ophthalmol. 2003;136(4):655-61. [PMID: 14516805] http://dx.doi.org/10.1016/S0002-9394(03)00388-X

35. Laidlaw DA, Tailor V, Shah N, Atamian S, Harcourt C. Validation of a computerised logMAR visual acuity measurement system (COMPlog): Comparison with ETDRS and the electronic ETDRS testing algorithm in adults and amblyopic children. Br J Ophthalmol. 2008;92(2):241-44. [PMID: 17993577] http://dx.doi.org/10.1136/bjo.2007.121715

36. Schubert MC, Migliaccio AA, Della Santina CC. Dynamic visual acuity during passive head thrusts in canal planes. J Assoc Res Otolaryngol. 2006;7(4):329-38. [PMID: 16810569] http://dx.doi.org/10.1007/s10162-006-0047-6

37. Schubert MC, Migliaccio AA, Clendaniel RA, Allak A, Carey JP. Mechanism of dynamic visual acuity recovery with vestibular rehabilitation. Arch Phys Med Rehabil. 2008;89(3):500-507. [PMID: 18295629] http://dx.doi.org/10.1016/j.apmr.2007.11.010 
38. Wiener-Vacher SR. Vestibular disorders in children. Int J Audiol. 2008;47(9):578-83. [PMID: 18821227]

http://dx.doi.org/10.1080/14992020802334358

39. Alvarez JC, Díaz C, Suárez C, Fernández JA, González del Rey C, Navarro A, Tolivia J. Neuronal loss in human medial vestibular nucleus. Anat Rec. 1998;251(4):431-38. [PMID: 9713981]

http://dx.doi.org/10.1002/(SICI)1097-

0185(199808)251:4<431::AID-AR2>3.0.CO;2-V

Submitted for publication December 17, 2010. Accepted in revised form June 20, 2011.
This article and any supplementary material should be cited as follows:

Rine RM, Roberts D, Corbin BA, McKean-Cowdin R, Varma R, Beaumont J, Slotkin J, Schubert MC. New portable tool to screen vestibular and visual functionNational Institutes of Health Toolbox initiative. J Rehabil Res Dev. 2012;49(2):209-20.

http://dx.doi.org/10.1682/JRRD.2010.12.0239

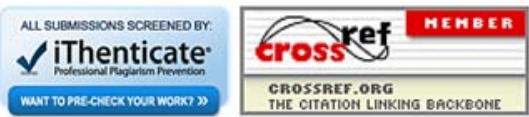

